\title{
Optimization of Anaerobic Granular Sludge by Removing Biogas
}

\author{
Guan Xijun", Xia Lijia \\ School of Environmental and Municipal Engineering, Qingdao University of Technology, Qingdao, China \\ Email address: \\ guanxj2004@qq.com (Guan Xijun) \\ ${ }^{*}$ Corresponding Author \\ To cite this article: \\ Guan Xijun, Xia Lijia. Optimization of Anaerobic Granular Sludge by Removing Biogas. American Journal of Water Science and \\ Engineering. Vol. 4, No. 3, 2018, pp. 75-79. doi: 10.11648/j.ajwse.20180403.13
}

Received: September 25, 2018; Accepted: October 10, 2018; Published: October 25, 2018

\begin{abstract}
The loss of granular sludge and acidification of sludge are common problems in highly efficient anaerobic reactors in operation at present, which results in that the reactors cannot reach the design COD removal rates. The problems must be solved for the widespread use of the highly efficient anaerobic reactors. There are many different studies on the formation and loss of granular sludge in an anaerobic reactor, however, the researchers ignore the effects of the gas-producing and biogas-off process of granular sludge on the formation mechanism of granular sludge. The sedimentation and buoyancy phenomena of the granular sludge cultured by self-made transparent reactor were observed in the lab and their relative velocities were measured by photos. Granular sludge and their biogas are coexistent, that is, any size particles of granular sludge can form high floating speed when granular sludge particles contain a lot of bubbles and the granular sludge particles can precipitate well when the air bubbles of granular sludge particles are released in time. So the water test on the sedimentation rate of granular sludge is insufficient. Unified consideration of granular sludge and biogas is beneficial to solve the loss of granular sludge and acidation of sludge in anaerobic reactors and to ensure the normal operation of anaerobic reactors. The biogas-off of biogas produced by anaerobic sludge has essential effects on the granulation of granular sludge. It is conducive to highly efficient anaerobic reactors and three phase separator to reconstruct.
\end{abstract}

Keywords: Biogas, Granular Sludge, Settlability, The Loss of Granular Sludge, Relative Density

\section{Introduction}

There are two focal problems in the practice operation of Up-flow Anaerobic Sludge Blanket and internal circulation reactors. One is that granular sludge is difficult to form in a reactor and it's operation depends on the flocculated sludge; the other is that the loss of granular sludge and acidification of sludge lead to decrease organic removal rate. The problem of the loss of granular sludge has caused widely concern, for example, gas tunnel was obstructed by extracellular polymer substances (EPSs) which led to the decreasing relative density and the floatation of granules [1], granular sludge with hydrophobic surface has strong adhesion with gas such as $\mathrm{CH}_{4}$, air bubbles cause the loss of granular sludge, the richer protein substrate is, the greater the loss of granular sludge is [2], the more complex the organic substrate is, the easier the flotation of sludge is [3], excessive hydraulic velocity caused the disintegration and the loss of granular sludge [4], the granular sludge had good sedimentation rate will also have the loss of granular sludge if biogas will not be separated from granular sludge [5], the relative density of granular sludge with a particle size of $1.0-2.5 \mathrm{~mm}$ is larger than 1 , while the relative density of smaller and the larger hollow granular sludge is less than 1 which is easy to float up and wash out of the reactor [6]. The relationship between granular sludge and anaerobic biogas was further studied by experiment and observation in the paper.

\section{Experiment and Observation}

\subsection{Experimental Device}

The experimental device is consistent with the diagram of patent application [7], as shown in Figure 1. It is constructed from a transparent plexiglass pipe with diameter of $200 \mathrm{~mm}$ 
and a height of $1500 \mathrm{~mm}$. Including: tank 1, inlet pipe 2 , inlet pump 3, check valve 4, sludge discharge horizontal pipe 5, valve 7 , outlet short pipe 8 , outlet pipe 9 , location water seal 10 , fixed pressure water seal 11 , air outlet short pipe 12 , air outlet pipe 13, bottom support 14, diffusion impeller 15, suction pipe 16 , bearing 17 , motor 18 , top support 19 , liquid level 20, gas chamber 21 and reaction zone 22.

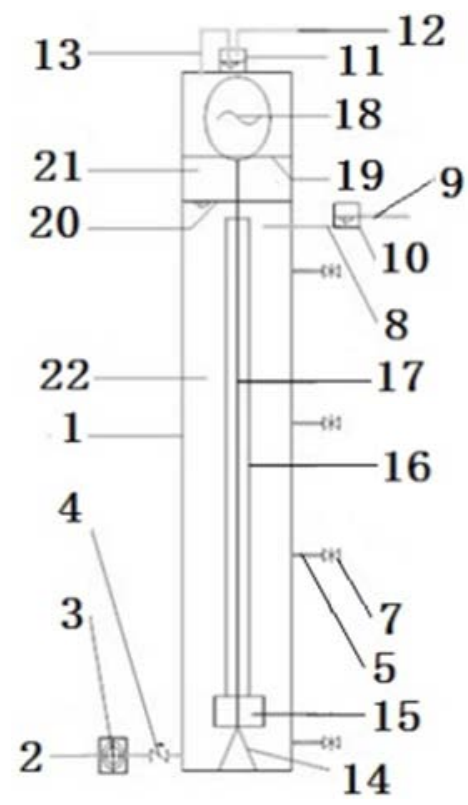

Figure 1. Experimental device for anaerobic.

\subsection{Test Condition}

Wastewater made from fruit simulates the industral waste water of fruit and vegetable canned beverage of Shandong happy family food co., LTD. The culturing and domesticating of anaerobic granular sludge use the anaerobic flocculated sludge of the Hebo-river wastewater treatment plant as seed. No else nutrients and additives were added during the experiment.

\subsection{Observation and Discussion of the Sludge Phenomenon of the Experimental Device}

Photo condition of experimental phenomena: the experimental reactor was operated to $45 \mathrm{~d}$, the COD volume load reached $4.5 \mathrm{~kg} / \mathrm{m}^{3} . \mathrm{d}$, the COD concentration of wasterwater was $5000 \sim 8000 \mathrm{mg} / \mathrm{L}$, the COD removal rate was $80 \sim 90 \%$, and the granular sludge was cultivated. Observation of the phenomena of experimental device can be seen: the sediment boundary surface is a churning state with a large number of overflowing air bubbles. There are a large number of rising and precipitating mud masses above the sediment boundary surface.

The granular sludge sampling in the measuring tube is shown in figure 2. It can be seen in figure 2 that a large amount of granular sludge has been formed in the reactor. The maturation of granular sludge was also confirmed by washing sludge.

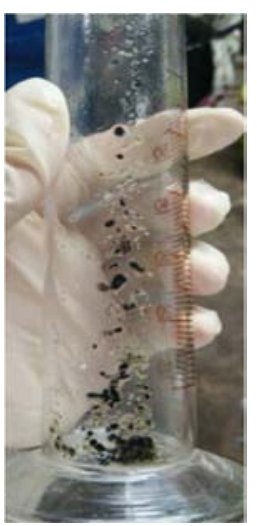

Figure 2. Granular sludge in the measuring.

The dynamic change of wasterwater and air and sludge in the reactor was not conducive to analysis by capture photos. In order to reflect the effect of air bubbles on granular sludge, the $26 \mathrm{~s}$ mobile phone video was recorded in the condition of the shooting height $10 \sim 16 \mathrm{~cm}$ of mobile phone (the experimental conditions are limited without any Foundation of China). The screenshots of the mobile phone video were shown in Figure 3 and Figure 4.

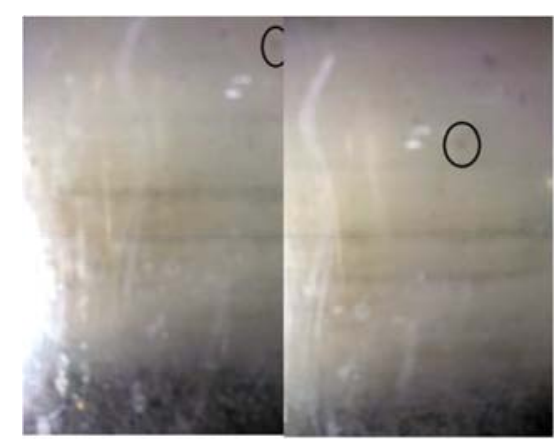

(a)

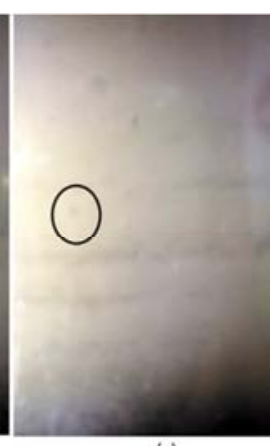

(c)
Figure 3. Sedimentation of granular sludge.

Figure 3 show the screenshots of the precipitating process of granular sludge. It can be seen from figure (a) and (b) and (c) that the main liquid level has been formed because of the precipitated sludge but a large amount of sludge is still sinking at a high rate of flow. The sinking sludge must be the degassing granular sludge after flotation. It can be measured that the sedimentation rate of the degassing granular sludge is about $65 \mathrm{~mm} / 3 \mathrm{~s}=78 \mathrm{~m} / \mathrm{h}$.
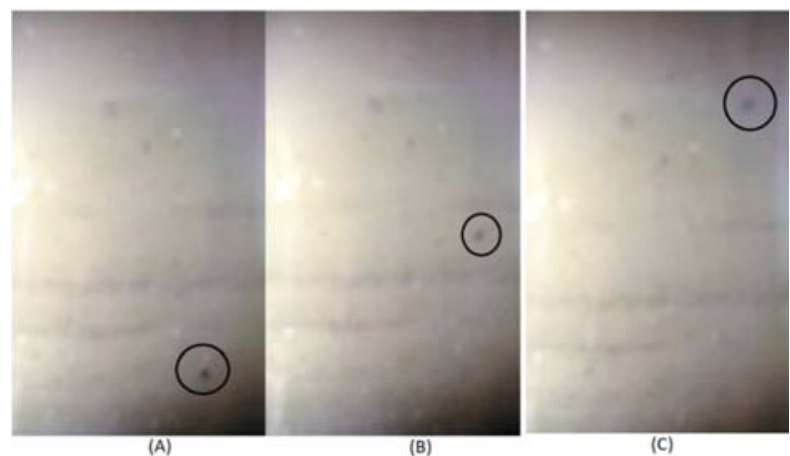

Figure 4. Flotation of granular sludge. 
Figure 4 shows the screenshots of the floating process of granular sludge. It can be seen from Figure 4 that granular sludge rises without hydraulic rising effect when this experimental device was observed. Sludge rising is not the concept of water washing. The granular sludge has a very high rising speed which is obviously the effects of upward air bubbles. It can be measured that the flotation rate of the granular sludge with bubbles is about $100 \mathrm{~mm} / 3 \mathrm{~s}=120 \mathrm{~m} / \mathrm{h}$.

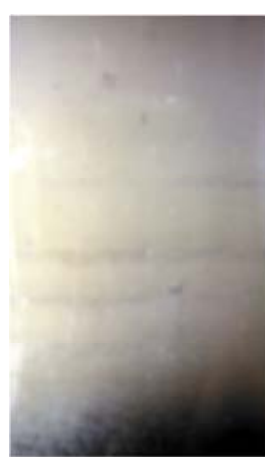

(A)

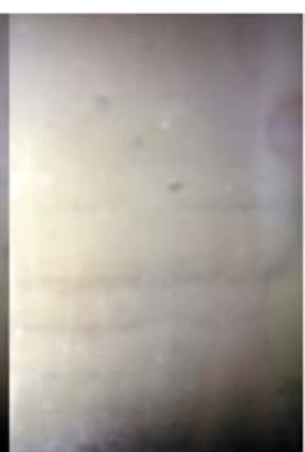

(B)

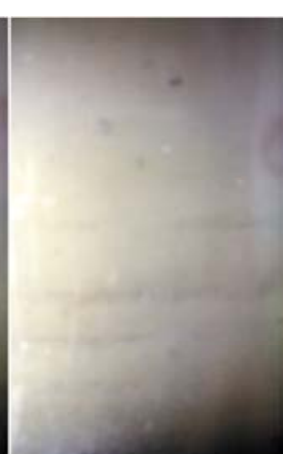

(C)
Figure 5. Flotation and sedimentation of granular sludge in $1 \mathrm{~s}$.

There are two particles of granular sludge sedimentation and one particle of granular sludge flotation in Figure 5. Figure 5 shows the difference of the right flotation and the left sedimentation of three particles of granular sludge in $1 \mathrm{~s}$. Rectangular particles float about $60 \mathrm{~mm}$ in $1 \mathrm{~s}$, the floating speed is about $60 \mathrm{~mm} / 1 \mathrm{~s}=216 \mathrm{~m} / \mathrm{h}$. Two sinking granular sludge particles are the left side of the floating granular sludge particles in the figure, and the sedimentation rate of two sinking granular sludge particles is significantly slower than the floating granular sludge particles.

The above experiments show that the flotation and sedimentation of the granular sludge particles depend entirely on how many bubbles in the particles. Once granular sludge particles contain a lot of bubbles, any size particles can form high floating speed. That is, the granular sludge particles containing bubbles will inestimably reduce their relative density. When the air bubbles of granular sludge particles are released in time, the granular sludge particles can precipitate well.

In the condition of no rising hydrodynamic velocity, the effect of biogas produced by granular sludge can be better explained in the experiments. It was fully proved by experiments that formation of anaerobic granular sludge and its retention in the reactor must be counted to their floating speed with bubbles. Scholars' views on the losses of granular sludge are hydrodynamic wash out, have no the detailed description of floating speed.

For example, the calculation method of sedimentation rate of granular sludge particles is the free fall terminal velocity of sludge particles in the tap water, which reflects indirectly the sedimentation rate of sludge particles [8]. The sedimentation distance and sedimentation time of granular sludge particles were measured in the organic glass tube filled with tap water, and their ratio was the sedimentation rate of granular sludge in water. The sedimentation rate is clearly measured in which granular sludge particles are an inhibited state and not a real growth state, and cannot truly represent the actual rate in the reactor. During a period of anaerobic stable operation, the sludge of granular sludge having a sufficient sedimentation rate will not flow out of the reactor [9]. High hydraulic shear forces produce the loss of granular sludge, the continuous increase of shear force resulted in the washing out of a large number of granular sludge and debris, which was the main cause of the reactor operation breakdown [10].

So it is necessary to discuss the effects of biogas production and release of granular sludge.

\section{Biogas Estimated Yield of Granular Sludge by Means of the Specific Methanogenic Activity (SMA)}

Sludge bubbles have seriously restricted the operation of anaerobic reactors. For example, 500 tons of granular sludge particles disappeared in 5 days in the IC reactor made by BEST environmental technology Co., LTD. in Xinxiang's antibiotic Co., Henan. The IC reactors made by Shandong huanlejia food CO, LTD. lost sludge and acidified severely in tank. The IC reactor treating slaughtering wasterwater of Shandong Xiantan CO, LTD. has not be debugged during 7 months. It was shown as Figure 6 that samplings of different heights of the UASB separate about 15-20 minutes, that reveals the effects of biogas.

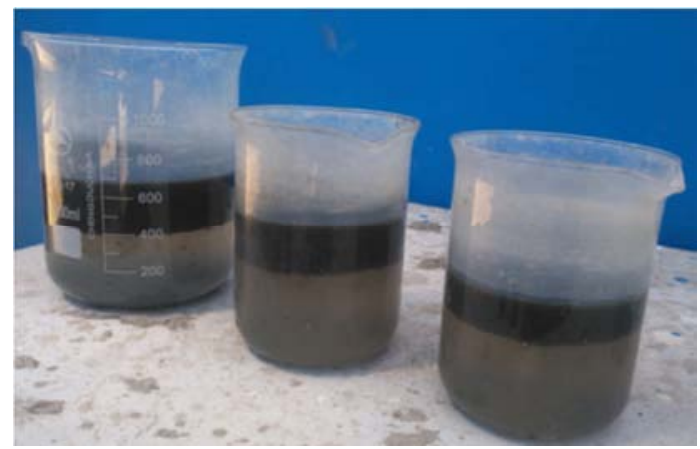

Figure 6. Separating samplings at different heights of a UASB.

Methane has a process releasing biogas in the floc. If the biogas does not detach from the floc in time, the relative density of the whole granular sludge will be less than that of water, thus causing loss of granular sludge. Researchers equate traditionally losses of granular sludge with washing out it, neglect the gas-producing process of granular sludge.

If the SMA is $0.3 \mathrm{~L} / \mathrm{g}$.d, the relative density of sludge is $1.05 \mathrm{~g} / \mathrm{mL}$, ignoring the volume of $\mathrm{CO}_{2}, \mathrm{H}_{2}$ and other gases:

VSS volume per gram is: $1 \mathrm{~g} \div 1.05 \mathrm{~g} / \mathrm{mL}=0.95$ $\mathrm{mL}=0.00095 \mathrm{~L}$.

Ratio of gas volume produced by 1 gVSS per day to VSS's: $0.3 \div 0.00095=316$.

The time interval of the same gas volume produced by 
granular sludge as itself: $86,400 \div 316=273 \mathrm{~s}$.

When the air bubble volume carried granular sludge reaches itself $5 \%$, the relative density is close to 1 , and the granular sludge may be washed out from the reactor. The time is $: 173 \times 5 \%=14 \mathrm{~s}$.

That is, if the gas capacity produced by granular sludge in $14 \mathrm{~s}$ cannot be discharged in time and retain in granular sludge, granular sludge can be floated and washed away. The three-phase separator is difficult to separate the biogas within the granular sludge from the granular sludge. The larger the losses of the gas-producing sludge of floc are, the more harmful the normal operation of anaerobic reactor is.

The experimental device is intermittently flooding water, mass transfer and degassing of granular sludge are realized by hydraulic water of mechanical intermittent stir, granular sludge does not depend on hydraulic screening and aerodynamic action like the conventional reactors, the precipitation interface of the anaerobic reactor looks like boiling. The sinking and floating of the granular sludge on the interface can better reflect the biogas production and release during no mechanical stir.

\section{Consistency and Improvement to the Existing theory}

\subsection{Diameter and Relative Density}

It has been confirmed by experiments that the relative density of granular sludge with particle size 1.0-2.5 is greater than 1 , while the relative density of granular sludge having a small or a hollowed-out large particle sizes is smaller than 1 . The less than 1 relative density of granular sludge is easy to float and wash out the reactor. The relative density of granular sludge does not change in the theory, the change is caused by air bubbles acting on granular sludge.

Assumption:

The minimum volume of air bubbles that can break away from granular sludge is fixed.

The specific surface area is proportional to the amount of adsorption to air bubbles, according with adsorption model.

Particle size $1.0 \mathrm{~mm}$ with $\mathrm{n}$ air bubbles has relative density 1 .

Granular sludge has an effective thickness (fixed film thickness).

The sludge particle of $1.0 \mathrm{~mm}$ has obviously the maximum surface area per unit weight and the maximum bubble $n$ within the particle size range of $1.0 \sim 2.5 \mathrm{~mm}$. The bigger the particle is, the bigger the relative density is. If the particle size range is in excess of $2.5 \mathrm{~mm}$ and the fixed film thickness, large particles appear the cavity occupied by bubbles. The bigger the particle, the bigger the hollow is. As the result, the relative density becomes lighter than 1 and granular sludge loses.

The particle size is less than $1.0 \mathrm{~mm}$ and its surface area is larger than that of $1.0 \mathrm{~mm}$. Because the adsorptive bubble's numbers per unit weight are larger than $n$, their relative density is less than 1, this result also in the loss of granular sludge.

\subsection{The Loss of Granular Sludge}

The starting loss of granular sludge can be explained that the relative density is less than 1 . The first, a large amount of newly formed small sludge particles adsorb too excessive air bubbles to be released. The second, sludge particles to be domesticated is suppressed and hollowed during starting.

The acidulated loss of granular sludge can be explained that granular sludge decomposes and combine bubbles with EPS, the relative density is less than 1 .

When the starch wastewater was treated by UASB reactor, it was found that the reason of the loss of granular sludge is excessive polysaccharides in EPS, its adhesion binds small bubbles so that the relative density of granular sludge decreases [11]. The hydraulic shear force can be too low to run in the culture process of granular sludge in anaerobic bottle, and the activity and stability of granular sludge obtained under the condition of $150 \mathrm{rpm}$ is not better than that obtained under the condition of $165 \mathrm{rpm}$ [12]. These phenomena and theories are consistent with the hypothesis in previous section.

\subsection{Hydrophobicity}

The gas producing and releasing of granular sludge are a process of constant replacement, that must have nanometer bubbles and nanometer gas layers which can persist for hours or even days [13]. Nano-bubbles and nano-gas layers make the sludge hydrophobic. proton migration and dehydration theory thinks that the translocation-dehydration and hydrophobicity of the cell surface are the beginning of cell adhesion [14].

\subsection{The Necessary and Insufficient Three-Phase Separator}

An important component of anaerobic reactors is the three-phase separator whose most patents were hold in China, Japan and the United States [15]. The structures of three-phase separator have impacting surface and necked-down section. The necked-down section obstructs the sludge to return to the main reaction area. The granule sludge particles with gas are degassed and precipitated by the extrusion changes of hydraulic conditions of the three-phase separator. Three-phase separators distinguish flocculated sludge with granular sludge separation, have inability to remove biogas in the granular sludge, cannot well cope with startup and restarting and impacting load and acidification.

\section{Conclusion}

The biogas and degas of the sludge play a great influence on their relative density, which is crucial to the generation and retention of granular sludge in the reactor. The biogas factors produced by the granular sludge can explain many theories and phenomena, which are helpful to solve the problems of the loss of granular sludge and acidification of sludge in the reactor. And the clear water precipitation experiment of granular sludge inhibits the gas producing and degassing, which cannot well represent the precipitation ratio 
of granular sludge in the reactor. Unified consideration of granular sludge and biogas is beneficial to solve the loss of granular sludge and acidation of sludge in anaerobic reactors and to ensure the normal operation of efficient anaerobic reactors. It is conducive to highly efficient anaerobic reactors and three phase separate reactor reconstruction.

\section{References}

[1] Lu Huifeng, Zheng Ping, Ji Qixing, et al. The structure, density and settle ability of anammox granular sludge in high-rate reactors $[\mathrm{J}]$. Bioresource Technology, 2012, 123: 312-317.

[2] Guo Xiao-lei, Hu Yong-you, Gao Kong-rong. Characters and mechanism of formation of anaerobic granular sludge [J]. Water \& Wastewater Engineering, 2000(01): 33-38.

[3] Hickey R F, Wu W M, Veiga M C, et al. Start-up, operation, monitoring and control of high-rate anaerobic treatment systems [J]. Water Science and Technology, 1991, 24(8): 207-255.

[4] FANG Xiao, JIANG Zhu-wu, ZHANG Ya-lei, YANG Hai-zhen. A Review of Characteristics and Granulation of Anaerobic Granular Sludge [J]. Jiangsu Environmental Science and Technology, 2005(04):44-46.

[5] Wang peng. Reasons and Control of anaerobic Granular Sludge Floating [J]. Journal of Taiyuan University, 2017, 35(03):45-48.

[6] Xu Fu. Full-scale cultivation of anaerobic granular sludge and its underlying mechanisms [D]. Jiangnan University, 2013.
[7] Guan Xijun. An anaerobic reactor [P]. Shandong: CN107758861A, 2018-03-06.

[8] Muda K, Aris A, Salim M R, et al. Development of granular sludge for textile wastewater treatment [J]. Water Research, 2010. 44(15): 4341-4350.

[9] Dong Chunjuan, Lu Bingnan. Characterisitic reaseach of ananerobic granules $[\mathrm{J}]$. Chinese Journal of Environmental Engineering, 2007(06):102-108.

[10] Bi Lei. Effects of Hydrodynamic Shear Force on Granular Sludge in Upflow Anaerobic Reactor [D]. Tsinghua University, 2007.

[11] Lu Xueqin, Zhen Guangyin, Adriana Ledezma Estrada, et al. Operation performance and granule characterization of upflow anaerobic sludge blanket (UASB) reactor treating wastewater with starch as the sole carbonsource [J]. Bioresource Technology, 2015, (180):264-273.

[12] Wang Liangjie. Experimental Research about Enhancements of Additives on Cultivating Anaerobic Granular Sludge [D]. China University of mining and technology, 2017.

[13] Zhao Binyu. Interfacial Properties of Nanobubbles at the Solid/liquid Interface Studied by Advanced Nano-Imaging [D]. The University of Chinese Academy of Sciences, 2015.

[14] Tay J H, Xu H L, Teo KC. Molecular mechanism of granulation. I: $\mathrm{H}^{+}$translocation-dehydration theory $[\mathrm{J}]$. Journal of Environmental engineering, 2000, 126(5):403-410.

[15] Wang Jing, Lu Wanxiang. review on the patent technology of Upflow anaerobic sludge blanket (UASB) [J]. Henan science and technology, 2017(11):90-91. 